\title{
Reducing PM Eddy Current Rotor Losses by Partial Magnet and Rotor Yoke Segmentation
}

\author{
Dominic A. Wills, Maarten J. Kamper
}

\begin{abstract}
In order to reduce the losses caused by eddy currents in the magnets and rotor yoke of a PM electric machine, a number of effective methods can be used. One method that imposes the least restrictions on machine performance is segmentation, which can be difficult to implement as magnets need to be cut, insulated and re-glued, which is a laborious and costly process. This paper presents methods of partial segmentation in magnets and the rotor yoke that aim to improve machine performance by reducing eddy current loss, while also suggesting realistic manufacturing possibilities. Results are obtained using analytical, numerical and experimental methods and good agreement is achieved.
\end{abstract}

Index Terms - permanent magnet, eddy current, losses, partial segmentation, rotor yoke, analytical, finite element.

\section{INTRODUCTION}

$\mathrm{E}$ ddy currents flowing in the rotor of a permanent magnet synchronous machine (PMSM) can be detrimental to a machine's performance. The ohmic power loss caused by circulating eddy currents is a source of inefficiency, and the heat produced in a solid rotor yoke can raise the magnet temperature above its recommended operating range causing partial, irreversible demagnetization.

In principle, a high proportion of eddy currents in the rotor are induced by asynchronously rotating stator MMF harmonics found with certain types of PMSM machine topologies. Methods to protect the rotor from the harmonics include closing the stator winding slots, including a steel retaining ring over the magnets, enlarging the air gap and segmenting the solid conducting regions.

A segmented region is comprised of smaller, insulated material pieces. Segmentation is the preferred method for reducing rotor losses as it has the least influence on the machine's performance. In this study, we consider the magnet and rotor regions to be radially segmented, i.e.: the incisions are made down the machine's axial length, as shown in Fig. 1. It has been shown that magnet segmentation has a marked effect on reducing eddy current magnet losses [1-3, 911,14]. Using analytical methods, these studies show the strong relationship between increased segmentation and reduction in magnet eddy current losses.

In [1], the author uses the stator current time harmonics to predict an inverse square relationship between the width of the magnet and the reduction in losses. In [4], [5] the model is expanded to include the moving MMF spatial harmonics in the stator winding. In [3], the model is extended to also include the reaction fields of the induced currents in the magnets and the yoke. The results of these methods agree that increased levels of segmentation lead to significantly lower magnet eddy current losses.

${ }^{\Phi}$ This work was supported in part by the South African National Antarctic Programme (SANAP) and the National Research Foundation (NRF) in South Africa.

D.A. Wills and M.J. Kamper are from the University of Stellenbosch, Private Bag X1, Matieland, 7602, South Africa (email: kamper@sun.ac.za)

.ac.za).
The segmentation manufacturing process includes cutting the magnet into equal-sized pieces after which the 'segments' are glued back together. This manufacturing process can prove to be costly and labour intensive, which prompts some manufacturers to avoid segmentation and find alternative means to reduce magnet losses. This study presents two alternative methods to full magnet segmentation (FMS) which aim to address some of the construction difficulties with FMS while still providing a considerable saving in rotor losses. Assumptions include ignoring the eddy current end effects as this model is computed in 2-D only.

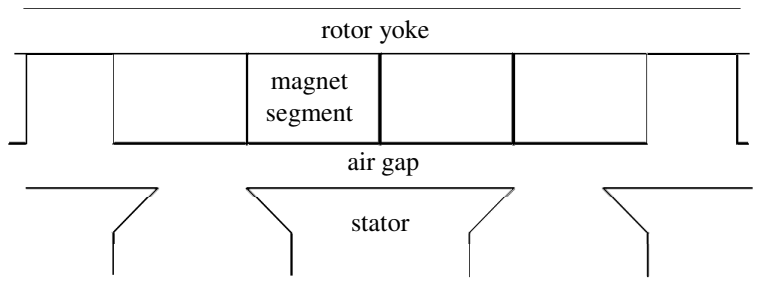

Fig. 1: A machine cross-section showing full magnet segmentation.

\section{ROTOR LOSS MODEL}

For the machine model, a radial flux PMSM machine is unrolled to create a linear machine model in the $(\mathrm{x}, \mathrm{y}, \mathrm{z})$ plane shown in Fig. 2. The co-ordinate axes are fixed to the moving rotor reference frame. A current sheet lies on the stator surface and represents either a balanced three phase stator winding flowing in the $\mathrm{z}$ direction or a collection of harmonics present due to the effect of slotting or a combination of both. The definitions of these current sheets can be found in [2] for the case of the three phase winding or in [6] for the case of stator slotting. In this study, for accuracy, the harmonic magnitudes are extracted from a single time step finite element solution on the stator surface and are represented as:

$$
\begin{gathered}
H_{(\mu)}\left(x_{s}, t\right)=\sum_{\mu=1}^{\infty} h_{(\mu)} \cos \left(\omega t-\pi \mu x_{s}\right), \\
H_{(\mu)}(x, y, t)=\sum_{\mu=0}^{\infty} \sum_{i=1,3,5 \ldots}^{\infty} \frac{\Lambda_{\mu}(\mathrm{y}) B_{r e m}}{2\left(\mu N_{s} \pm i \frac{N_{p}}{2}\right)} \cdot \cos \left[\left(\mu N_{s}\right.\right. \\
\left.\left. \pm i \frac{N_{p}}{2}\right) x_{r}+\mu N_{s} \omega_{s} t\right]
\end{gathered}
$$

for the current harmonics and slotting harmonics respectively, where $\mu$ is the spatial harmonic, $h(\mu)$ is the magnitude of the $\mu^{\text {th }}$ spatial harmonic, $\omega$ is the angular frequency of the $\mu^{\text {th }}$ harmonic, $\omega_{s}$ is the synchronous rotor angular velocity, $x_{s}$ is the stator $x$ variable, $x_{r}$ is the rotor reference frame $x$ variable, $N_{s}$ is the number of slots, $N_{p}$ is the number of poles, $B_{r e m}$ is the flux density for a uniform air gap, $\Lambda(y)$ is the permeance variation due to slotting and $t$ is time. The higher order time harmonics are ignored as we assume purely sinusoidal stator current waveforms. 


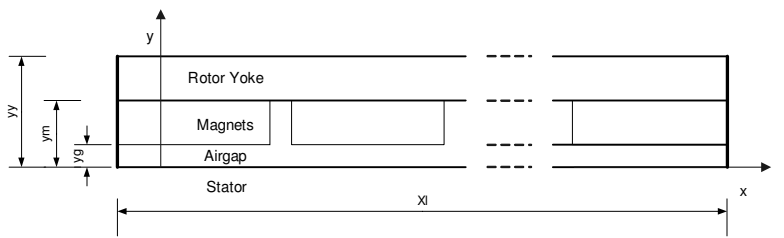

Fig. 2: The linear machine model shown in the $\mathrm{x}, \mathrm{y}$ plane.

The machine is divided into regions which describe the rotor yoke, magnets, air gap and stator as seen in Fig. 2. Each region has different properties of permeability and conductivity, leading to different solution constants for the magnetic vector potential function in each.

The transfer function to convert the stationary stator current loading harmonics onto the rotating rotor reference frame is defined as

$$
\omega=\omega_{s}-2 \pi \mu n,
$$

where $n$ is the rotor speed. Note than when $\mu$ is equal to the torque-producing working harmonic, the speed of this harmonic relative to the rotor is zero as the two harmonics are rotating synchronously in the air gap. All other current loading harmonics rotate asynchronously in the rotor causing eddy currents in the magnets and rotor yoke. In order to determine the eddy current function in the magnets, the magnetic vector potential, $A$ is used. We use Poisson's Equation to solve for $\mathbf{A}$,

$$
\nabla^{2} \boldsymbol{A}-j \omega \mathrm{K} \mu \boldsymbol{A}=0
$$

Using the method of separation of variables, which assumes that the overall function can be written as a product of two functions of $x$ and $y$ respectively, we assume the form:

$$
A(x, y)=A(x) A(y)
$$

This gives Poisson's Equation in the form of

$$
\frac{d^{2} A(x)}{d x^{2}} \cdot \frac{1}{A(x)}+\frac{d^{2} A(y)}{d y^{2}} \cdot \frac{1}{A(y)}=0
$$

Various solutions are available for this differential equation. Ignoring eddy currents, the solution best fitting this model is:

$$
A(x, y)=\left(C_{y} e^{\gamma y}+D_{y} e^{-\gamma y}\right) e^{-\alpha x}
$$$$
\text { where } \quad \gamma^{2}=\alpha^{2}+j \omega \mathrm{k} \mu \text {, and } \alpha=\mu \pi
$$

The general solution for the magnetic vector potential in each region is shown in (7). The next step is to define the exact constants for each region, which is done by defining the boundary conditions at the edges of the three regions studied. There are two conditions to be applied at the boundaries of each region:

$$
\begin{gathered}
B_{n, k}=B_{n, k+1} \\
H_{t, k+1}-H_{t, k}=A_{k}
\end{gathered}
$$

The boundary conditions stipulate that the normal value of flux density should be continuous across regions and that changes in tangential field intensity are created by the presence of current loading. The stator surface vector potential is defined by the stator loading current sheet. The air gap, magnets and yoke interfaces have continuous normal flux and have zero current loading, and the vector potential of the outer yoke boundary is set to zero. The detailed calculation of these constants can be found in [3]. One now has the complete, analytical solution for the magnetic vector potential in the areas of interest in the rotor of the machine. The particular quantity of interest for calculation of ohmic loss in the magnets is the eddy current density. The direction of the eddy current is assumed to be in the z-direction only and is calculated by:

$$
J(x, y)=-j \omega A(x, y)+\operatorname{grad} \varnothing
$$

where $\Phi$ is a constant in the $(x, y)$ plane. The eddy currents flowing in a solid conductor must have an equal and opposite return path i.e. taking the direction into account, currents in a conductor must sum to zero. The constant grad $\Phi$ term is used to offset residual DC current. Thus, we have:

$$
\begin{gathered}
J_{s e g}(x, y)= \\
\sum_{i=0}^{N p-1} \sum_{k=0}^{N s s-1} J(x, y) \int_{0}^{h_{m}} \int_{\frac{2 \pi}{N p} i+\frac{\tau k}{N s s}}^{\frac{2 \pi}{N p} i+\frac{\tau(k+1)}{N s s}} J(x, y) d x d y
\end{gathered}
$$

It should be noted at this juncture, that the change in current density due to the post process condition imposed in (12) will affect the field solution calculated from (9). This possible effect is ignored in the analytical calculations, but is taken into account in the FE analysis through the setting up of boundary conditions. The total power is calculated as:

$$
P_{\text {ave }}=\frac{l_{m}}{2 \sigma} \overline{J_{\text {seg }}(x, y) \cdot J_{\text {seg }}(x, y)^{*}}
$$

\section{A. Partial Magnet Segmentation}

The first proposed method to reduce the magnet loss is shown in Fig. 3(a) called single-sided partial magnet segmentation (SS-PMS). This technique is similar to full segmentation; however the penetration of the incision is less than $100 \%$. This technique ensures that the magnet stays in one piece during manufacture, which avoids complications with trying to bond fragmented magnet pieces. The second alternative shown in Fig. 3(b) is called double-sided partial magnet segmentation (DS-PMS). This technique uses the concept of SS-PMS, except that non-aligned cuts are made from either face of the magnet. This method also ensures that the magnet stays in one piece during the process of segmentation, but the advantage is that both sides of the magnet are segmented. These two techniques of PMS seek to alleviate some of the construction challenges experienced with FMS, while still aiming to achieve good eddy current loss reduction. The extent to which these loss reduction benefits are realized, forms the subject of this study.

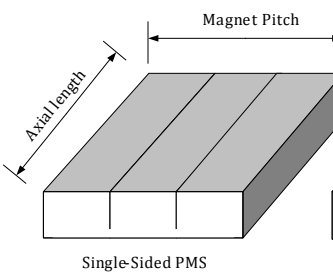

(a)

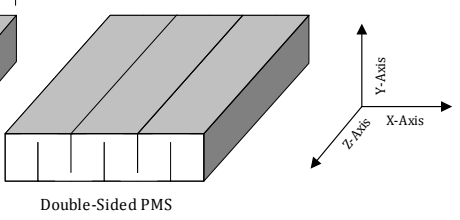

(b)
Fig. 3: Cross sections of (a) single-sided and (b) double-sided partially segmented magnets, showing incisions having partial penetration only.

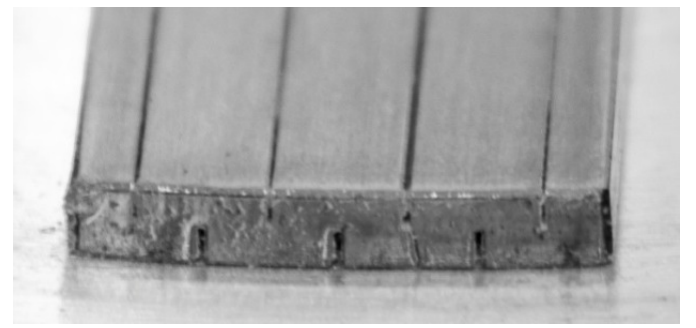

Fig. 4: Double-sided partially segmented magnet with incisions cut from both sides of the magnet and $50 \%$ penetration. 


\section{B. Partial Rotor Yoke Segmentation}

When voltages are induced in a solid conductor due to magnetic flux pulsations, the induced eddy current magnitudes are determined by the resistivity and the geometric dimensions of the solid in which they flow. In order to reduce losses in a solid rotor yoke, one must find a way to increase the resistivity without changing the material. This section describes a method of increasing the resistivity 'seen' by the eddy currents called partial rotor yoke segmentation (PRYS).

PRYS is a technique where the solid yoke is finely cut along the surface adjacent to the magnets as shown in Fig. 5. The aim of the cut is to create isolated conducting regions interrupting the eddy current flow. The depth of the cut is called the segmentation penetration depth and an optimum value depends on the conductivity, permeability, and the wavelength of the destructive field harmonics in the rotor. In many cases a segmentation penetration depth value much less than the thickness of the yoke can be selected.

The model used in this work is an analytical model of the stator MMF harmonics in the machine. The model includes the effect of the eddy current reaction field; however it neglects the effect of non-linear saturation properties of the steel rotor yoke. What is aimed for is a good machine model that can give insight and understanding into the causes of the magnet loss to help guide the design process. Finite element (FE) analysis is used for accurate prediction of results and comparison.

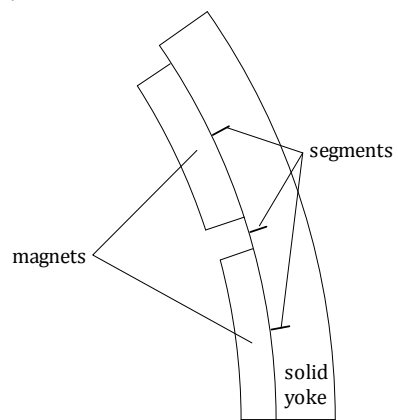

Fig. 5: Solid yoke partial segments positioned on the magnet side of the solid rotor yoke.

\section{RESULTS}

In order to make a definitive comparison of the methods of partial segmentation, three methods of generating results were used. The analytical model was used to gain qualitative results and understanding, the FE method was used to gain accurate results especially when materials with non-linear properties are used. A test machine was also built with a variety of rotors in order to validate the results obtained. The machine used was an outer rotor, 40 pole, 48 slot, nonoverlap, single layer wound, $15 \mathrm{~kW}$ PM machine seen in Fig. 6 , with its machine data shown in Table I.

To ensure consistency in the analytical model, FEA results are used to define the stator surface current sheet. The magnitude of each field harmonic is extracted from the stator surface FE calculation using a Fourier transformation, and eddy currents are computed using the harmonic magnitude and frequency. This frequency is calculated using Table II. The model was used to calculate the eddy currents in the magnet in order to compute the total magnet loss which was used to compare the novel proposed methods of partial segmentation.

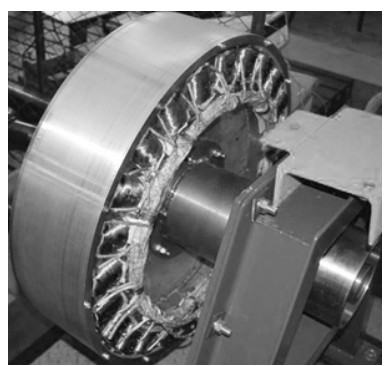

(a)

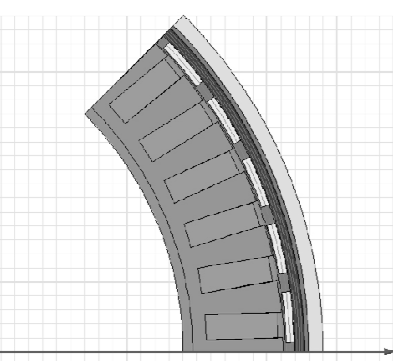

(b)
Fig. 6: (a) PM test machine and (b) layout of FE machine model.

\section{A. Single-sided Partial Magnet Segmentation}

The cross sectional power density function in the magnet is shown in Fig. 7 for varying degrees of SS-PMS. The segmented portion of the magnet has a marked effect on the magnet loss density in that region, while the unsegmented portion of the magnet predictably returns a loss profile expected in a solid magnet. It is clear from the power density function in Fig. 7 that when considering SS-PMS, segmentation in the air gap-facing half of the magnet is most effective as this targets the half of the magnet with the higher power density. The overall loss improvement with SS-PMS calculated numerically and analytically is shown in Fig. 8.

\section{B. Double-sided Partial Magnet Segmentation}

The power loss density benefit associated with using DSPMS is shown in Fig. 9. To avoid alignment of the magnet segments, the front and back of the magnet have differing degrees of segmentation, which explains the small discontinuity in magnet loss density appearing halfway through the magnet. This unequal segmentation underpins the reason why DS-PMS marginally outperforms the FMS. The relationship between magnet loss and segmentation is shown in Fig. 10 for a magnet with DS-PMS. It is clear that there is a significant reduction in magnet loss with increasing level of segmentation. The comparison of DSPMS with FMS can be further seen in Fig. 11, where they achieve very similar loss reduction properties with increasing segmentation.

TABLE I

MACHINE DimENSIONS AND PHOTOGRAPH OF THE PERMANENT Magnet SYNCHRONOUS MACHINE THAT WAS USED FOR TESTING AND ANALYSIS.

\begin{tabular}{|l|l|}
\hline Machine Parameter & Dimension \\
\hline Stator inner diameter & $247 \mathrm{~mm}$ \\
\hline Stator outer diameter & $311.5 \mathrm{~mm}$ \\
\hline Rotor outer diameter & $326.75 \mathrm{~mm}$ \\
\hline Stack length & $100 \mathrm{~mm}$ \\
\hline Rotor yoke thickness & $7.25 \mathrm{~mm}$ \\
\hline Magnet pitch & $0.73 \%$ \\
\hline Magnet thickness & $6 \mathrm{~mm}$ \\
\hline Rated speed & $150 \mathrm{rpm}$ \\
\hline Air gap length & $2 \mathrm{~mm}$ \\
\hline Coil width & $18 \mathrm{~mm}$ \\
\hline Tooth width & $11.1 \mathrm{~mm}$ \\
\hline
\end{tabular}


TABLE II

THE TABLE DESCRIBES FIELD HARMONIC ORIGIN AND SHOWS THE HARMONIC FREQUENCY CALCULATION.

\begin{tabular}{|c|c|c|}
\hline $\begin{array}{l}\text { Harmonic } \\
\text { source }\end{array}$ & Harmonic Definition & $\begin{array}{c}\text { Harmonic frequency } \\
\text { relative to rotor }\end{array}$ \\
\hline $\begin{array}{c}\text { Stator } \\
\text { winding }\end{array}$ & $\begin{array}{c}\mu=n \cdot \operatorname{scm}\left(N_{p}, N_{s}\right) \\
n=1,5,7,11,13 \ldots\end{array}$ & $\omega=\frac{2 \pi n_{s}}{60}\left(\frac{N_{p}}{2}-\mu\right)$ \\
\hline Static Magnet & $\begin{array}{l}\mu=\frac{n \cdot N_{p}}{2} \\
\mathrm{n}=1,3,5 \ldots\end{array}$ & $\omega=0$ \\
\hline Slotting & $\begin{array}{c}\mu=n N_{s}+i \frac{N_{p}}{2} \\
n=1,2,3 \ldots \\
i= \pm 1,3,5 \ldots\end{array}$ & $\omega=\frac{i}{a b s(i)} \frac{2 \pi n_{s} N_{s} R_{s}}{60}$ \\
\hline
\end{tabular}

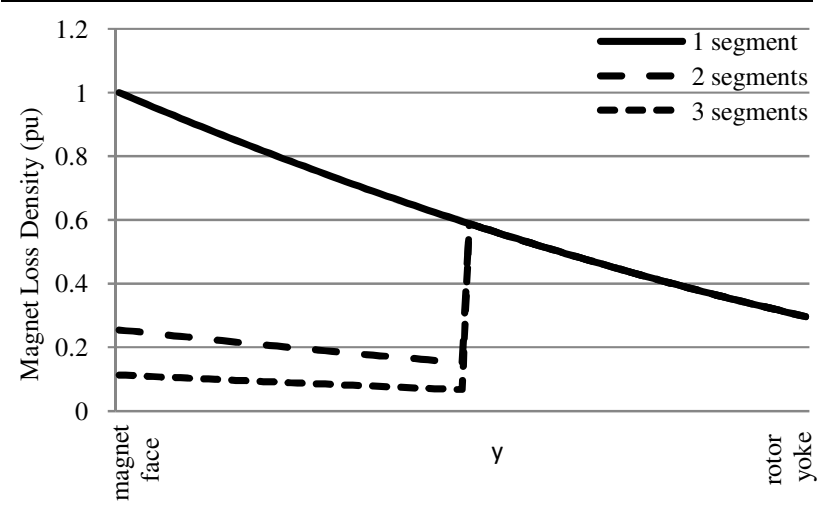

Fig. 7: Magnet loss change from the magnet face to the rotor yoke in a magnet with SS-PMS and $50 \%$ penetration.

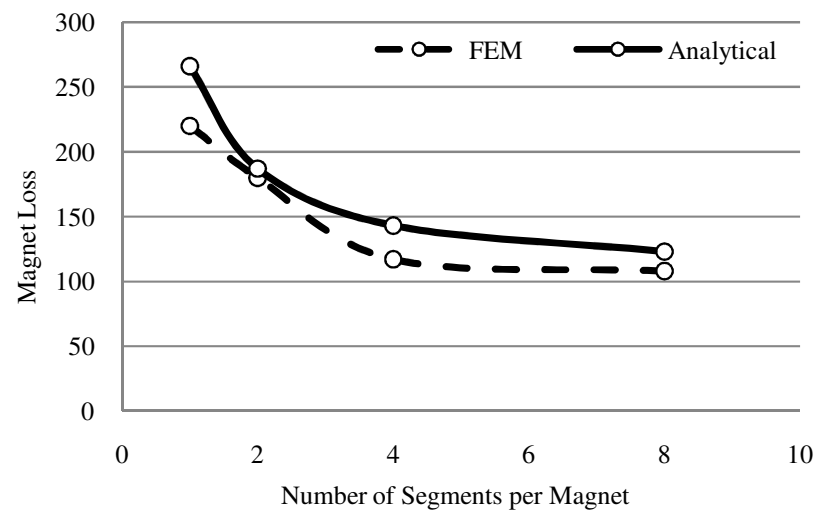

Fig. 8: Analytical and FE calculated magnet loss versus number of magnet segments with SS-PMS.

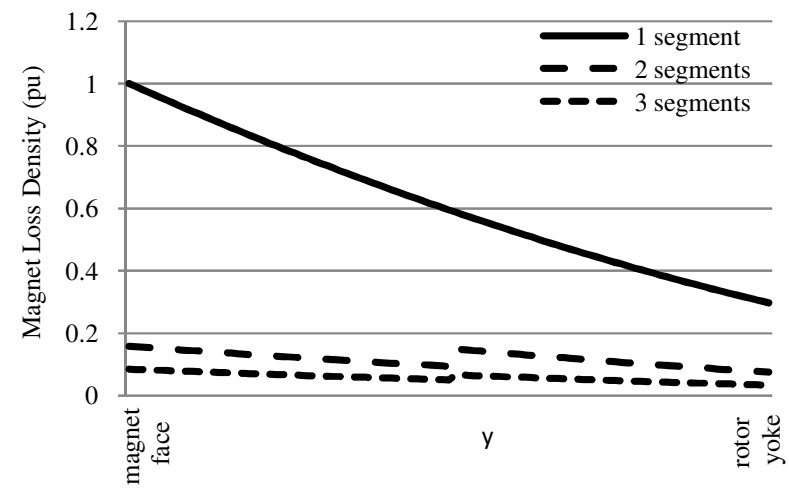

Fig. 9: Magnet loss change from the magnet face to the rotor yoke in a magnet with DS-PMS and $50 \%$ penetration.

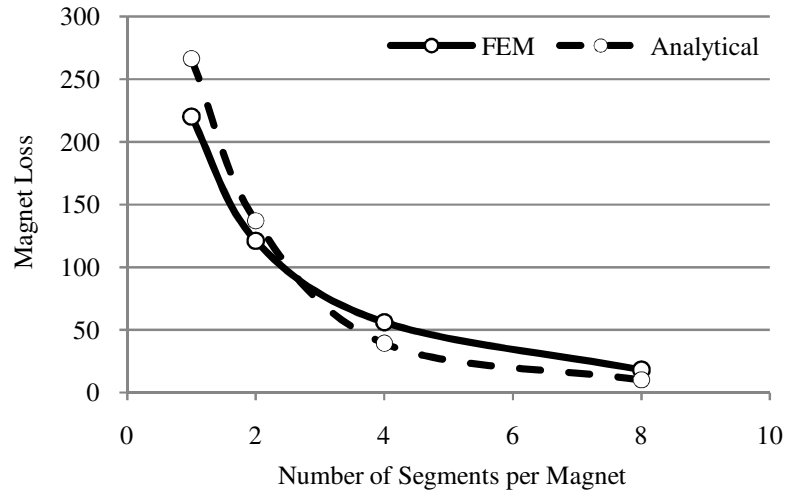

Fig. 10: Analytical and FE calculated magnet loss versus number of magnet segments with DS-PMS

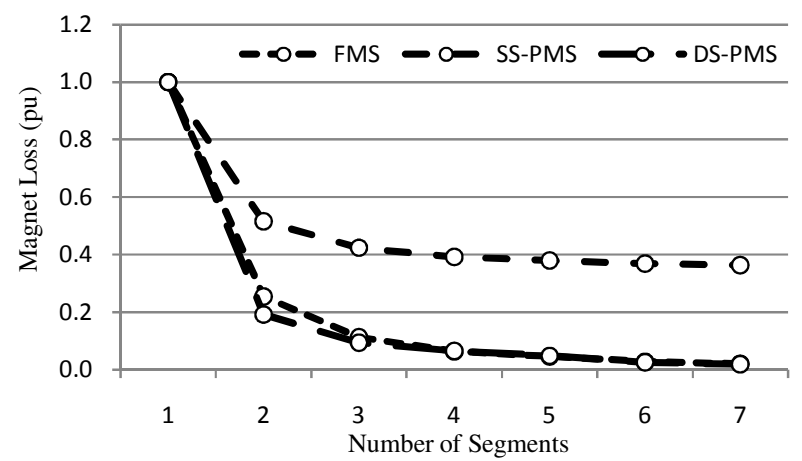

Fig. 11: Analytically calculated magnet loss versus number of segments for the three methods of magnet segmentation.

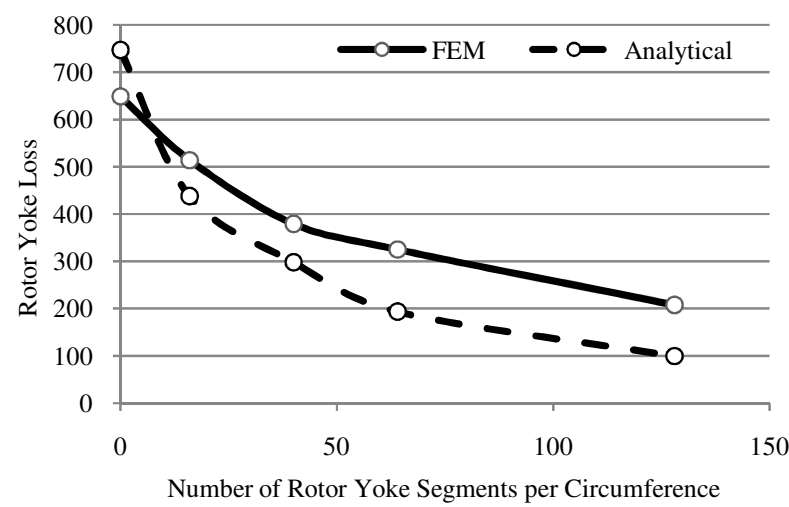

Fig. 12: Analytical and FE calculated rotor yoke loss versus level of rotor yoke partial segmentation and $17.5 \%$ penetration.

\section{Partial Rotor Yoke Segmentation}

The effect of the number of partial rotor yoke segments in minimizing the rotor yoke solid loss is shown in Fig. 12. The agreement between the analytical and FE method results is slightly compromised by the effect of varying permeability in the steel. Nevertheless, a good performance return is at higher levels of segmentation. Including 128 incisions around the inner yoke diameter of the machine with a penetration of $17.5 \%$ of the $7.25 \mathrm{~mm}$ yoke thickness shows a solid loss reduction (FE calculated) of $68 \%$ in the yoke. Using the calculation method in the Appendix, this improvement translates into a $24.8^{\circ} \mathrm{C}$ reduction in the yoke temperature and an overall efficiency improvement of $2.94 \%$.

The power density graph in Fig. 13 shows the intensity of the power loss due to eddy current flow in the cross section of 
the rotor yoke calculated analytically. It is clear in this study that due to the skin effect in the steel, the eddy currents only flow within $\pm 30 \%$ of the steel closest to the magnet interface. Thus, it is not necessary to segment the rotor yoke with a penetration greater than this percentage.

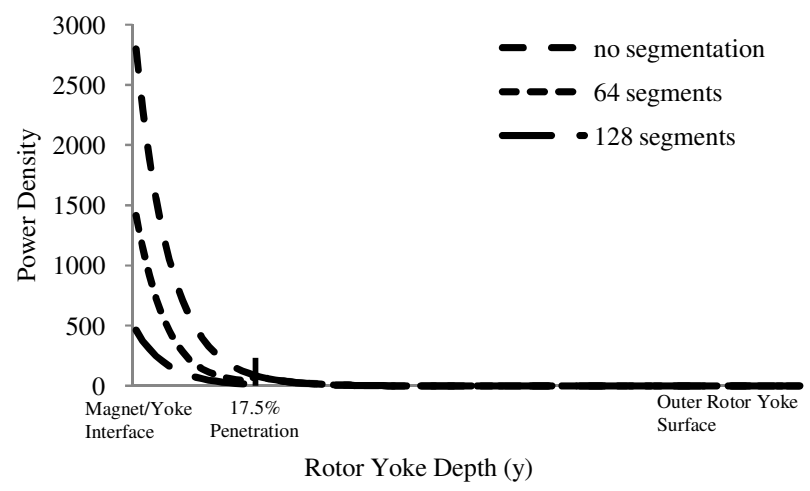

Fig. 13: Power density in the rotor yoke due to eddy currents flow.

\section{Comparison with measured results}

The magnet eddy current losses are measured using a technique described in the Appendix. The losses are measured from the machine in Fig. 6a that was built with two separate rotors to test the effect of DS-PMS. The first rotor contained solid magnets while the second rotor contained DSPMS magnets with four segments per magnet as shown in Fig. 4. The test machine is operated in generator mode with no electrical load as this eliminates the copper loss in the machine and also much of the stator core loss. TABLE III gives a comparison between the total rotor loss in the two rotors as measured and as calculated using analytical and FE methods. The loss improvement column shows the performance benefit of introducing DS-PMS over using solid magnets.

\section{TABLE III}

TOTAL ROTOR POWER LOSS OF THE TEST MACHINE AT 150R/MIN AT NO LOAD WITH SOLID AND DS-PMS MAGNETS.

\begin{tabular}{|c|c|c|c|}
\hline & Solid Magnets (W) & $\begin{array}{c}\text { DS-PMS } \\
\text { Magnets (W) }\end{array}$ & $\begin{array}{c}\text { Loss } \\
\text { Improvement (W) }\end{array}$ \\
\hline FEM & 755 & 591 & 164 \\
\hline Analytical & 781 & 554 & 227 \\
\hline Measured & 807 & 607 & 200 \\
\hline
\end{tabular}

\section{E. Overall Comparison}

The main FE calculated results of the test machine at full load are given in Table IV, i.e. to compare the various loss reduction techniques side-by-side. There can be confidence of their accuracy given the close agreement of measured and FE calculated results. These techniques include single and double sided partial magnet segmentation and partial rotor yoke segmentation.

If the best case scenario is extracted from Table IV, it can be deduced that implementation of DS-PMS with four segments per magnet and coupled with PRYS with 128 segments, would yield an efficiency improvement of $4.1 \%$ and a temperature reduction of $34.7^{\circ} \mathrm{C}$; which would have the $\mathrm{PM}$ rotor running at approximately $75^{\circ} \mathrm{C}$ at full load, which is well within the operating temperature range of the magnets.
TABLE IV

EFFECT OF VARIOUS LOSS REDUCTION TECHNIQUES ON THE FULL LOAD FE CALCULATED ROTOR EDDY CURRENT LOSSES OF THE TEST MACHINE.

\begin{tabular}{|c|c|c|c|c|}
\hline Technique & $\begin{array}{c}\text { Number of } \\
\text { Segments }\end{array}$ & $\begin{array}{l}\text { Magnet/yoke } \\
\text { Loss (W) }\end{array}$ & $\begin{array}{c}\% \text { Loss } \\
\text { Reduction }\end{array}$ & $\begin{array}{c}\text { Temperature } \\
\text { Reduction }\left({ }^{\circ} \mathrm{C}\right)\end{array}$ \\
\hline \multirow{3}{*}{ 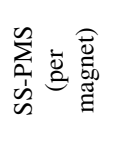 } & 1 & 229 & 0 & 0 \\
\hline & 4 & 138 & 39.7 & 5.11 \\
\hline & 8 & 118 & 48.4 & 6.23 \\
\hline \multirow{3}{*}{ 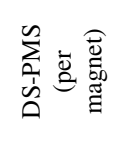 } & 1 & 229 & 0 & 0 \\
\hline & 4 & 53 & 76.9 & 9.88 \\
\hline & 8 & 32 & 86 & 11 \\
\hline \multirow{3}{*}{ 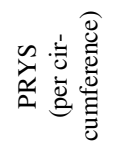 } & 1 & 649 & 0 & 0 \\
\hline & 64 & 325 & 50 & 18.2 \\
\hline & 128 & 208 & 68 & 24.8 \\
\hline
\end{tabular}

\section{CONCLUSION}

It is clear from analytical calculations that the rotor eddy current density decays exponentially with distance from the stator surface in both the magnets and the rotor yoke. SSPMS makes use of this property by implementing segmentation only on the stator-facing half of the magnet where the losses are more concentrated. Given the relative ease with which SS-PMS can be implemented and the low extra cost incurred, the price to performance ratio makes SSPMS a very attractive option. DS-PMS shows that even a relatively low level of segmentation can realize a significant reduction in losses. It can be concluded that DS-PMS is at least as effective as FMS at reducing magnet losses, while also being considerably easier to implement.

The exponential decay of the current density can be seen more clearly demonstrated in the rotor yoke due to the higher conductivity and permeability values. The rotor yoke eddy current density of the tested machine is shown to vanish completely within the first $30 \%$ of the yoke from the statorfacing side. This phenomenon explains the success of partial rotor yoke segmentation, as only the eddy current conducting area of the yoke needs to be segmented. This is quite achievable with minimal sacrifice in machine performance.

\section{REFERENCES}

[1] H. Polinder and M.J. Hoeijmakers, "Eddy-current losses in the segmented surface-mounted magnets of a PM machine", IEE Proc.Electr. Power Appl., Vol. 46, No. 3, May 1999

[2] Z.Q. Zhu, K. Ng, N. Schofield and D. Howe, "Improved analytical modelling of rotor eddy current loss in brushless machines equipped with surface mounted permanent magnets", IEE Proc.-Electr. Power Appl., Vol. 151, No. 6, November 2004

[3] R. Nuscheler, "Two-Dimensional Analytical Model for Eddy Current Loss Calculation in the Magnets and Solid Rotor yokes of Permanent Magnet Synchronous Machines", Proceedings of the 2008 international conference on Electric Machines, Paper ID 1095

[4] Kais Atallah, David Howe, Philip H. Mellor, and David A. Stone, "Rotor Loss in Permanent-Magnet Brushless AC Machines", IEEE Transactions On Industry Applications, Vol. 36, No. 6, November/December 2000

[5] Hiroaki Toda, Zhenping Xia, Jiabin Wang, Kais Atallah, David Howe, "Rotor Eddy-Current Loss in Permanent Magnet Brushless Machines", IEEE Transactions On Magnetics, Vol. 40, No. 4, July 2004

[6] D. Howe, Z.Q. Zhu, "Instantaneous Magnetic Field Distribution in Brushless Permanent Magnet dc Motors", Part III: Effect of Stator Slotting, IEEE Transactions on Magnetics, Vol. 29, No.1, January 1993 
[7] Nicola Bianchi, Michele dai Pre, Luigi Alberti, Emanuele Fornasiero, "Theory and design of Fractional-Slot PM Machines", September 2007.

[8] Dahaman Ishak, Z. Q. Zhu, David Howe, "Eddy-Current Loss in the Rotor Magnets of Permanent-Magnet Brushless Machines Having a Fractional Number of Slots Per Pole", IEEE Transactions on Magnetics, vol. 41, no. 9, September 2005

[9] Yasuaki Aoyama, Koji Miyata, Ken Ohashi, "Simulations and Experiments on Eddy Current in Nd-Fe-B Magnet", IEEE Transactions on Magnetics, Vol. 41, No. 10, October 2005

[10] H. Polinder, M.J. Hoeijmakers, M. Scuotto, Eddy-Current Losses in the Solid Back-Iron of PM Machines for different Concentrated Fractional Pitch Winding, IEEE International Electric Machines \& Drives Conference, 2007. IEMDC '07, Volume 1, 3-5 May 2007, p. 652 - 657

[11]Jason D. Ede, Kais Atallah, Geraint W. Jewell, Jiabin Wang, David Howe, "Effect of Axial Segmentation of Permanent Magnets on Rotor Loss in Modular Permanent-Magnet Brushless Machines", IEEE Transactions on industry applications, Vol. 43, no.5, September/October 2007.

[12]Miroslav Markovic, Yves Perriard, "Analytical Solution for Rotor Eddy-Current Losses in a Slotless Permanent-Magnet Motor: The Case of Current Sheet Excitation", IEEE Transaction on Magnetics, Vol. 44, No.3 March 2008.

[13]Nicola Bianchi, Michele dai Pre, Luigi Alberti, Emanuele Fornasiero, "Theory and design of Fractional-Slot PM Machines", September 2007.

[14]Peter Sergeant, Alex van den Bossche, "Segmentation of Magnets to Reduce Losses in Permanent-Magnet Synchronous Machines", IEEE Transactions on Magnetics, Vol. 44 No. 11, November 2008.

[15] Nicola Bianchi, Emanuele Fornasiero, "Impact of MMF Space Harmonic on Rotor Losses in Fractional-Slot Permanent-Magnet Machines", IEEE Transactions on energy conversion, Vol. 24, No.2, June 2009.

\section{BIOGRAPHIES}

Dominic A. Wills was born in Cape Town, South Africa in 1981. He graduated from University of Cape Town with a BSc (Eng) in 2004 and $\mathrm{MSc}$ (Eng) in 2006. He then graduated with $\mathrm{PhD}$ in Electrical and Electronic Engineering from University of Stellenbosch in 2010. He is currently working in electric vehicle technology at Optimal Energy in Cape Town. His research interests lie within the field of permanent magnet generator design, analytical and numerical computation and with particular reference to rotor efficiency.

Maarten J. Kamper received the M.Sc. (Eng.) degree in 1987 and the Ph.D. (Eng.) degree in 1996 both from the University of Stellenbosch, Stellenbosch, South Africa.

He has been with the academic staff of the Department of Electrical and Electronic Engineering, University of Stellenbosch since 1989, where he is currently a Professor of electrical machines and drives. His research interests includes computer-aided design and the control of reluctance, permanent magnet and induction electrical machine drives with applications in electric transportation and renewable energy. Prof. Kamper is a South African National Research Foundation Supported Scientist and a Registered Professional Engineer in South Africa.

\section{APPENDIX}

The no load rotor loss measurement is done by comparing the losses in three different PM rotors. The rotors are identical in geometry, but differ in terms of magnet segmentation and rotor yoke core material as:

PM rotor 1: solid magnets and solid mild steel rotor yoke;

PM rotor 2: DS-PMS and solid mild steel rotor yoke;

PM rotor 3: DS-PMS and laminated rotor yoke.

For each of the three PM rotors the input shaft power, $P_{i n}=$ $\omega_{s} \tau$, and the steady state outer rotor temperature, $T_{r o}$, are measured, with the PM machine each time at no load and driven at a speed of $150 \mathrm{r} / \mathrm{min}$. Assuming no hysteresis loss in the PM rotor, the first equation for the total rotor eddy current loss, $P_{e r}$, is given by

$$
P_{e r}=P_{i n}-P_{\text {loss }}
$$

where $P_{\text {loss }}=P_{w f}+P_{s}$, i.e. equal to the wind and friction losses plus the core and winding eddy current losses in the stator.

In a second equation for $P_{e r}$ Newton's thermal law of cooling is considered. In the thermal model two assumptions are made. Firstly it is assumed that heat transfer takes place only through convection, thus heat transfer through radiation is ignored. Secondly, heat transfer to ambient via moving air in the air gap is ignored. With these two assumptions $P_{e r}$ can be expressed approximately as

$$
\begin{aligned}
P_{e r} & =\frac{d Q}{d t} \\
& \approx h_{o} A_{o}\left(T_{r o}-T_{a}\right)+h_{i} A_{i}\left(T_{r i}-T_{a s}\right)
\end{aligned}
$$

where $Q$ is the heat energy, $h_{o}$ and $h_{i}$ are the outer and inner rotor surface heat transfer coefficients, $A_{o}$ and $A_{i}$ are the outer and inner rotor yoke surface areas, $T_{r o}$ and $T_{r i}$ are the measured outer and inner rotor surface temperatures and $T_{a}$ and $T_{s}$ are the ambient and stator temperatures respectively. It was found during measurements that $T_{s} \approx T_{a}$ and that $T_{r i} \approx T_{r o}$, so that (15) can be simplified as

$$
\begin{aligned}
P_{e r} & \approx\left(h_{o} A_{o}+h_{i} A_{i}\right)\left(T_{r o}-T_{a}\right), \\
& =h A\left(T_{r o}-T_{a}\right)
\end{aligned}
$$

From (14) and (16) three equations can be obtained for the three PM rotor measurements assuming $P_{\text {loss }}$ stays constant, namely as

$$
\begin{aligned}
& P_{i n(1)}-P_{\text {loss }} \approx h A\left(T_{r o}-T_{a}\right)_{1} \\
& P_{i n(2)}-P_{\text {loss }} \approx h A\left(T_{r o}-T_{a}\right)_{2}, \\
& P_{i n(3)}-P_{\text {loss }} \approx h A\left(T_{r o}-T_{a}\right)_{3}
\end{aligned}
$$

where subscripts 1, 2 and 3 refer to PM rotor 1, PM rotor 2 and PM rotor 3 respectively as described above. From (17) on average the constants $P_{\text {loss }}$ and $h A$ can be determined. With these constants known $P_{e r}$ can be determined by (14) or (16). Obviously, from (17) the power loss difference DS-PMS makes can also be measured. 